\title{
STEERING ENTROPY CHANGES AS A FUNCTION OF MICROSLEEPS
}

\author{
Amit Paul ${ }^{1}$, Linda Ng Boyle ${ }^{1,3}$, Erwin R. Boer ${ }^{4}$, Jon Tippin ${ }^{2}$, Matthew Rizzo ${ }^{1,2,3}$ \\ ${ }^{1}$ College of Engineering \\ ${ }^{2}$ College of Medicine \\ ${ }^{3}$ Public Policy Center \\ University of Iowa \\ Iowa City, Iowa, USA \\ E-mail: linda-boyle@uiowa.edu \\ ${ }^{4}$ LUEBEC
San Diego, California, USA
}

\begin{abstract}
Summary: This study aimed to assess steering entropy as a measure of decrements in driving performance caused by microsleeps. Microsleeps are brief, unintended episodes of loss of attention that last 3-14 seconds. These episodes, which are frequent in drivers with sleep disorders, can be long enough to impact steering performance and are particularly disruptive when driver action is imperative, as when driving around curved highway segments. Steering entropy is a driver-centered performance measure that can detect drivers' corrective responses to situations when the vehicle state falls outside the driver's expectations. This study tests the hypothesis that steering entropy is an indicator of increased erratic steering behavior during microsleep episodes in drivers with obstructive sleep apnea/hypopena syndrome (OSAHS). Twenty-four drivers with OSAHS were used in this study and their electroencephalography (EEG) defined microsleep (cases) and non-microsleep episodes (crossover control) were compared using a case-crossover method. The performance measure, steering entropy, was calculated from a time-series history of steering angle data. Steering entropy was compared for each microsleep in the three-second interval both immediately preceding and immediately following each microsleep. Results showed that steering entropy was higher on curves during microsleeps and post microsleeps when compared to straight road segments and the no-workload baseline condition. This suggests that steering entropy can capture erratic steering behavior, allowing us to better understand how drivers correct for previous steering errors.
\end{abstract}

\section{INTRODUCTION}

Motor vehicle crashes cause great suffering and the national economic costs exceed $\$ 200$ billion (Blincoe et al., 2002). Many of these crashes are caused by sleepy drivers (Horne \& Reyner, 1995; Leger, 1994). Sleep disorders, such as obstructive sleep apnea/hypopnea syndrome (OSAHS), which affects 5 to 10 million Americans (National Commission on Sleep Disorders Research, 1993), elevate the risk of sleep-related vehicular crashes. Patients with OSAHS have recurrent episodes of reduced or absent airflow during sleep, caused by intermittent obstruction of the nasopharynx and/or oropharynx (American Thoracic Society, 1994). Patients typically exhibit symptoms of loud snoring, intermittent gasping, fragmented and non-restorative sleep periods and excessive daytime sleepiness. 
An important step toward reducing injury and damages caused by sleep-related crashes is a better understanding of the effects of sleep disorders on driver performance and safety errors so that an objective and reliable criteria for detecting and alerting drowsy drivers can be developed. In this vein, we examined the steering control of drivers with OSAHS using a measure known as Steering Entropy (SE), as shown in Nakayama et al. (1999). We tested the hypothesis that SE can be used as an indicator of erratic steering behavior in the context of microsleep episodes in drivers with OSAHS.

Microsleeps are indicator of excessive daytime sleepiness and have been associated with poor simulated driving performance (Risser et al., 2000). The hypothesis is that a microsleep increases the potential for deviation from the lane because steering is temporarily neglected. This potential is especially strong when steering is neglected in a curve or during periods in which the vehicle is perturbed by lateral disturbances, such as strong wind gusts, road canter, or bumps and ruts in the road surface. During microsleeps, these disturbances cause the vehicle to drift towards the lane edge; drivers respond after the microsleep with a corrective steering action that is more aggressive than what is observed during wakeful driving. SE is particularly designed to identify and quantify these larger and faster than normal corrective actions and is thus expected to provide a selective and sensitive metric to identify microsleeps and quantify their consequences for how comfortable drivers are with the lane deviation resulting from the microsleep.

\section{METHODOLOGY}

\section{Subjects}

The subjects in the study were 24 legally licensed adult drivers between the ages of 30 and 65 (mean age $=47.9, \mathrm{SD}=9.93$ ) with medically diagnosed OSAHS. There were 12 women (mean age $=49.5$ ) and 12 men (mean age $=46.3$ ). Subjects were paid $\$ 50$ for their participation.

\section{Experimental Procedure}

Driving behavior was tested in driving scenarios implemented on SIREN (Simulator for Interdisciplinary Research in Ergonomics and Neuroscience) (Rizzo et al., 2000). Each participant drove on three laps of a simulated 2-lane highway with interactive traffic. Each lap consisted of curved road segments with 600 meter radii (3 left turn, 3 right turn). Participants were instructed to drive at speeds below the 55-mph speed limit and it took approximately 60 minutes to complete a test. No lateral disturbances other that the curves were implemented.

\section{Performance Measures}

Microsleeps. Microsleeps were defined as follows: (1) episodes of 3-14 seconds duration, (2) absence of alpha activity $(8-13 \mathrm{~Hz})$ alone or, if some alpha activity present, frequency slowed $>1$ $\mathrm{Hz}$ compared to baseline, (3) increase in theta activity (4-7 Hz), (4) absence of movement or blink artifact obscuring EEG, and (5) attenuation of alpha not explained by the normal blocking response caused by eye opening (Harrison \& Horne, 1996; Robinson \& Guilleminault, 1999). The EEG was interpreted by a neurologist certified by the American Board of Sleep Medicine. Recordings were made using a Neurofax EEG-2100 (Nihon Khoden Corp, Tokyo) with a 200-Hz sampling rate. 
Steering Entropy. Steering entropy (SE) is a measure of randomness in a driver's steering control. SE is higher when drivers make larger erratic steering movements, indicating potentially unsafe behavior (e.g., overcorrecting for unanticipated road changes). The driver's steering input produces an output that defines the trajectory of the simulator vehicle on the virtual roadway. During distraction or sleepiness (e.g., during a microsleep), the vehicle may drift unexpectedly outside the driver's lateral comfort zone. Feedback of this unexpected drift prompts the driver to make an aggressive steering correction. These larger and faster steering actions make the steering profile less predictable, producing greater SE. The quantitative SE calculation used in this study was adapted from Nakayama et al. (1999) and was defined as

$$
S E=\sum_{i=1}^{9}\left(-p_{i} \cdot \log \left(p_{i}\right)\right) \text { where } \mathrm{i}=1 \ldots 9
$$

where $p_{i}$ is the proportion of steering prediction errors, $e(n)$, falling into one of nine bins, $i$, created based on the $90^{\text {th }}$ percentile value from a steering prediction error distribution of a baseline segment of the drive. For this study, the baseline segment for each subject was chosen as the first 5-8 minutes of the drive, where workload was at a minimum (i.e., no known microsleeps and the road segment was straight). The prediction errors, $e(n)$, as defined by Nakayama et al. (1999) is :

$$
e(n)=\theta_{a}(n)-\theta_{p}(n)
$$

where $\theta_{a}(n)$ is the actual steering angle and $\theta_{p}(n)$ is the predicted steering angle at time $n$ and is based on a second-order Taylor expansion series (Weisstein, 2002). Also, the steering angle data used in this study were transformed from a frequency of $60 \mathrm{~Hz}$ to $6.67 \mathrm{~Hz}$ to account for the lowest sampling frequency that can justifiably be used to represent a human operator's control.

A pilot analysis showed the feasibility for using SE as a performance measures in drivers with OSAHS. Figure 1 shows a broader distribution of steering prediction errors in a driver with OSAHS compared to a driver without any sleep disorder. The broader distribution of steering prediction error suggested that OSAHS drivers would show higher SE than drivers without a sleep disorder.

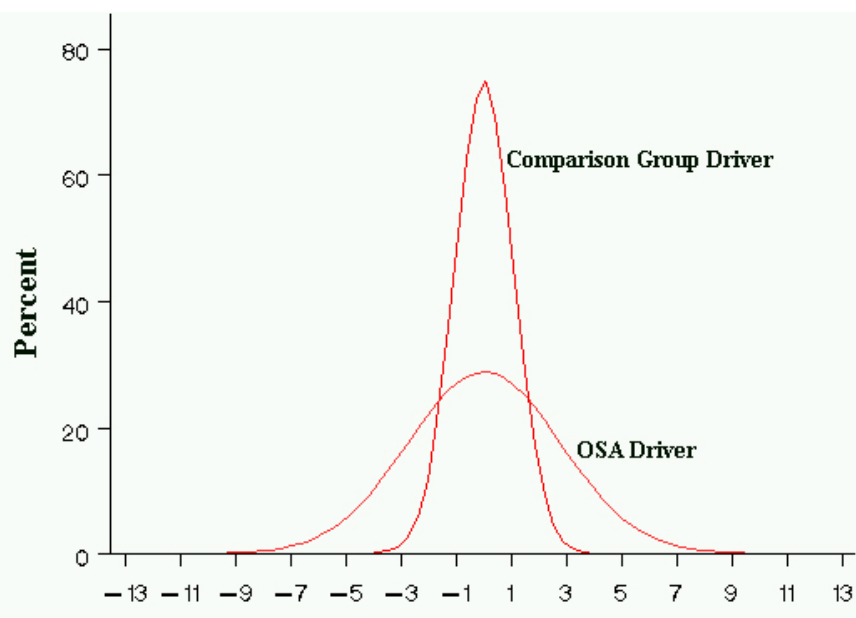

Steering Prediction Error, e(n)

Figure 1. Distribution of steering prediction error for an OSAHS driver and a non-OSAHS driver 


\section{Experimental Design}

We used a case-crossover design to assess the transient changes in behavior associated with microsleeps. A case-crossover design provides a means of looking at the effects of a condition during periods when the condition is present and when it absent (Marshall, 1993) and has been successfully used to assess associations between cellular telephone calls and motor vehicle collisions (Redelmeier \& Tibshirani, 1997). This design is also appropriate for evaluating the effects of microsleeps when they are present as opposed to absent during a drive. To evaluate driving performance during microsleeps, we identified control (non-microsleep) episodes during the drive that could be matched to the case (microsleep) episodes. Figure 2 shows that a 2:1 matched approach was used to compare driver behavior during the periods when microsleeps occurred, with behavior during the three-second periods immediately preceding (pre) and immediately following (post) each microsleep. The three-second duration was chosen because it was comparable to the minimum duration for a microsleep and minimized the chances of encountering confounding driving and environmental effects.

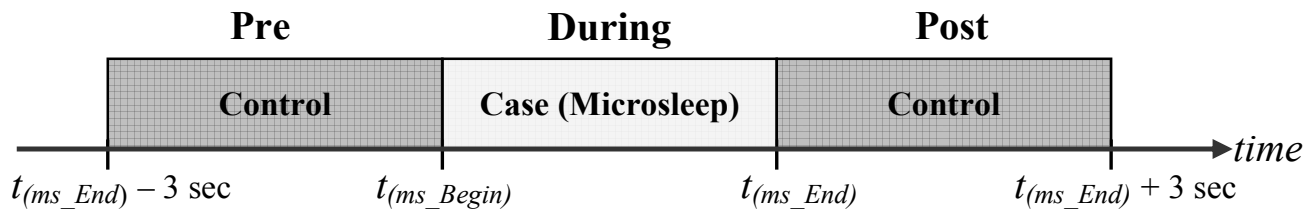

Figure 2. A 2:1 matched design case-crossover study

A repeated measures analysis of variance (ANOVA) was used to analyze the differences among the independent variables. There were two independent variables used in this study: microsleep periods (pre, during, post), and road segments (straight, curve). Intercept and the number of microsleep episodes were included as random effects. Given that SE did not follow a normal distribution, an exponential transformation was done in order to meet the normality assumptions of ANOVA. A nonparametric test (such as Kruskal-Wallis) was not used because this assumes that the response variable does not follow any distribution, nor can the measures be transformed in any way. That is not the case with our dependent variables.

\section{DATA ANALYSIS AND RESULTS}

Steering entropy during microsleep periods was 0.48 and post-microsleep periods were 0.45 . These values are fairly close to the SE under conditions of no workload (SE =0.471) as mentioned by Nakayama et al. (1999; see Table 1). There were 150 microsleep events used for this study. Each OSAHS driver had, on average, 8 microsleep events (s.d. $=9.01$, range 1 to 39) with the number of microsleeps per driver following a negative binomial distribution (Figure 3 ).

Table 1. Steering entropy at pre, during and post microsleep periods

\begin{tabular}{lccc}
\hline \multirow{2}{*}{ Steering Entropy } & \multicolumn{3}{c}{ Microsleep episodes } \\
\cline { 2 - 4 } & Pre & During & Post \\
\hline Mean & 0.40 & 0.48 & 0.45 \\
Std. Deviation & 0.05 & 0.05 & 0.05 \\
\hline
\end{tabular}




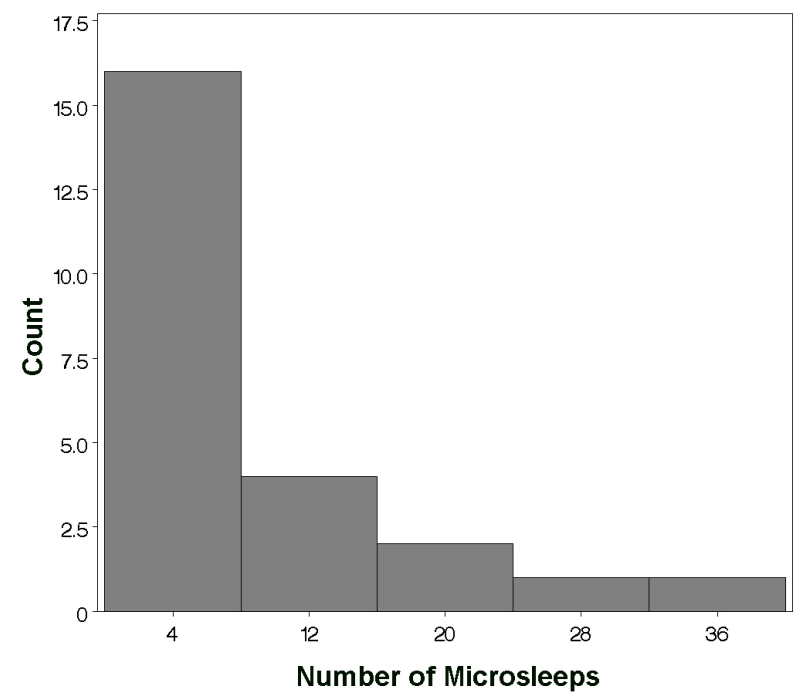

Figure 3. Distribution of number of microsleeps per driver

The repeated measures mixed-effects ANOVA showed that SE differed among the three microsleep periods (pre, during and post $)(\mathrm{F}(2,336)=5.49, \mathrm{p}<0.005)$ and was higher during the microsleep episodes than during the pre- and post-microsleep periods. There was also a significant SE effect based on the road segments $(\mathrm{F}(1,156)=7.17, \mathrm{p}<0.01)$. Figure 4 shows that $\mathrm{SE}$ increased on curved roads compared to on straight segments. However, there was no interaction effect $(\mathrm{F}(2,336)=1.88, \mathrm{p}>0.05)$. Drivers who had microsleeps on curved road segments exceeded Nakayama's safety thresholds for steering entropy $(\mathrm{SE}=0.471)$ both during and post, possibly due to increased workload while driving on curves.

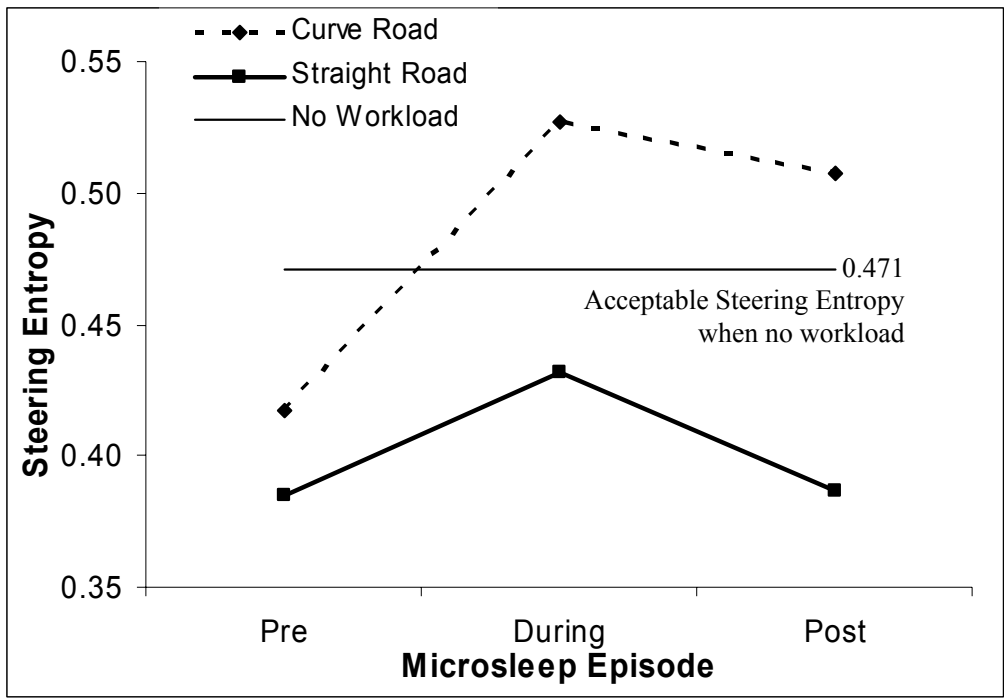

Figure 4. Comparison of effect microsleep on SE after adjusting for microsleeps on curves 


\section{DISCUSSION}

The concept of entropy discussed in this paper has been used in assessing human workload in the driving (Östlund et al., 2004) and non-driving domains (Goodrich et al., 2004). In the Östlund et al. (2004) study, SE was used to investigate the effects of task workload and in-vehicle information systems on driving. In Goodrich et al. (2002), behavioral entropy was used to estimate human workload in human-robot interactions. Therefore, there appears to be great promise in using SE to evaluate performance during a microsleep.

Nakayama et al (1999) used SE to assess workload effects over minutes-long intervals. In this study, SE calculations were based on microsleep durations, which were based on a smaller time scale (from 3-14 seconds). We used SE to detect changes in driver steering control during microsleep periods and on different roadway types, in line with our introductory hypothesis that SE would provide an indicator of erratic steering control in drivers with OSAHS during microsleeps.

Drivers are constantly judging the situation ahead and applying small, smooth, steering adjustments (Nakayama et al., 1999) by turning the steering wheel in small increments. When a vehicle enters a curve, the driver makes small steering angle adjustments to keep inside the lane. Steering predictability decreases as drivers apply more corrective maneuvers. This decreased steering predictability results in increased SE, reflecting the increased workload associated with driving on curves as opposed to straight segments of highway. Of note, SE does not decrease during microsleep episodes as might be expected during periods of inaction. Rather, entropy increases during microsleep episodes as expected during periods of inattention or distraction. This reflects the fact that during microsleeps, drivers are in a transitional phase of consciousness between wakefulness and sleep. They are not fully asleep and can continue to engage in normaland abnormal-appearing patterns of motor behavior, as has been confirmed from our video reviews of driver activities. These behaviors include steering and accelerator pedal control movements, which may be under automatic control of attention, as well as more erratic behaviors associated with loss of motor control (such as "head nodding" due to loss of cervical muscle control). Overall, their behavior during microsleeps leads to higher SE.

On straight segments, drivers with OSAHS also have higher SE during microsleeps. However, as Figure 4 shows, SE on straight segments is still lower than the SE in a no-workload baseline condition. Similarly, SE also increases on curves when a microsleep is present. This higher SE on curves indicates that drivers with OSAHS make large steering corrections post microsleep. In real life, these corrections may or may not be sufficient to recover control of a vehicle before a lane deviation error or a crash results. Note that not all microsleeps lead to a lane deviation.

Future research should further address the relationships between SE and other more conventional driving performance measures that have been used in safety research, such as lane deviation, time to lane crossing, and acceleration variation. It should also focus on using physiologic evidence of changes in driver state, such as the occurrence of microsleeps, to aid countermeasures to sleepy driving. This study demonstrates suggests that microsleeps can provide a real-time marker for abnormal driver physiology that might be used to trigger driver alerting and warning devices on curved as well as straight road segments. 


\section{REFERENCES}

American Thoracic Society. (1994). Sleep apnea, sleepiness, and driving risk. American Journal of Respiratory and Critical Care Medicine, 150: 1463-1473.

Blincoe, L. J., Seay, A., Zaloshnja, E., Miller, T. R., Romano, E., Luchter, S., et al. (2002). The economic impact of motor vehicle crashes, 2000. Washington, DC: National Highway Traffic Safety Administration.

Goodrich, M. A., Boer, E. R., Crandall, J. W., Ricks, R. W., \& Quigley, M. L. (2004). Behavioral Entropy in Human-Robot Interaction. Paper presented at Proceedings of PERMIS, Gaithersburg, Maryland, Aug 24-26.

Harrison, Y., \& Horne, J. A. (1996). Occurrence of "microsleeps" during daytime sleep onset in normal subjects. Electroencephalogr Clin Neurophysiol, 98(5): 411-416.

Horne, J. A., \& Reyner, L. A. (1995). Sleep related vehicle accidents. British Medical Journal, 310(6979): 565-567.

Leger, D. (1994). The Cost of Sleep-Related Accidents - A Report for the National-Commissionon-Sleep-Disorders-Research. Sleep, 17(1): 84-93.

Marshall, R. J. (1993). Analysis of case-crossover designs. Statistics in medicine, 12(24): 23332341.

Nakayama, O., Futami, T., Nakamura, T., \& Boer, E. R. (1999). Development of a steeringentropy method for evaluating driver workload. Paper presented at SAE International Congress and Exposition, Detroit, Michigan, USA.

National Commission on Sleep Disorders Research. (1993). Wake Up America: A National Sleep Alert. Rockville, MD: US Dept of Health and Human Services.

Östlund, J., Nilsson, L., Carsten, O., Merat, N., Jamson, H., Jamson, S., et al. (2004). Deliverable 2 - HMI and Safety-Related Driver Performance: Human Machine Interface And the Safety of Traffic in Europe.

Redelmeier, D. A., \& Tibshirani, R. J. (1997). Association between cellular-telephone calls and motor vehicle collisions. New England Journal of Medicine, 336(7): 453-458.

Risser, M. R., Ware, J. C., \& Freeman, F. G. (2000). Driving simulation with EEG monitoring in normal and obstructive sleep apnea patients. Sleep, 23(3): 393-398.

Rizzo, M., McGehee, D., \& Jermeland, J. (2000). Design and installation of a driving simulator in a hospital environment. In: K. Brookhuis, D. DeWaard \& C. Weikert (Eds.), Human System Interaction: Education, Research and Application in the 21st Century. Maastricht, The Netherlands: Shaker Publishing: 69-77

Robinson, A., \& Guilleminault, C. (1999). Obstructive sleep apnea syndrome. In: S. Chokrovery (Ed.), Sleep Disorders Medicine: Basic Science, Technical Considerations and Clinical Aspects (2nd ed.). Boston: Butterworth-Heinemann: 241-254.

Weisstein, E. (2002). CRC Concise Encyclopedia of Mathematics (2nd ed.). Boca Raton, FL: CRC Press. 